\title{
Der Effekt Selbstregulierten und Forschenden Lernens auf kooperative und individuelle Lernprozesse in der Lernwerkstatt Religion Plural (LeRP)
}

Kramer, Kathrin [Hrsg.]; Rumpf, Dietlinde [Hrsg.]; Schöps, Miriam [Hrsg.]; Winter, Stephanie [Hrsg.]: Hochschullernwerkstätten - Elemente von Hochschulentwicklung? Ein Rückblick auf 15 Jahre Hochschullernwerkstatt in Halle und andernorts. Bad Heilbrunn : Verlag Julius Klinkhardt 2020, S. 368-378. - (Lernen und Studieren in Lernwerkstätten)

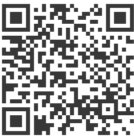

Quellenangabe/ Reference:

Balzer, Linda: Der Effekt Selbstregulierten und Forschenden Lernens auf kooperative und individuelle Lernprozesse in der Lernwerkstatt Religion Plural (LeRP) - In: Kramer, Kathrin [Hrsg.]; Rumpf, Dietlinde [Hrsg.]; Schöps, Miriam [Hrsg.]; Winter, Stephanie [Hrsg.]: Hochschullernwerkstätten - Elemente von Hochschulentwicklung? Ein Rückblick auf 15 Jahre Hochschullernwerkstatt in Halle und andernorts. Bad Heilbrunn : Verlag Julius Klinkhardt 2020, S. 368-378 - URN:

urn:nbn:de:0111-pedocs-212289 - DOI: 10.25656/01:21228

https://nbn-resolving.org/urn:nbn:de:0111-pedocs-212289

https://doi.org/10.25656/01:21228

in Kooperation mit / in cooperation with:

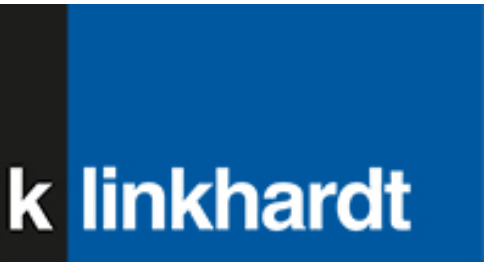

http://www.klinkhardt.de

\section{Nutzungsbedingungen}

Dieses Dokument steht unter folgender Creative Commons-Lizenz: http://creativecommons.org/licenses/by-nc-sa/4.0/deed.de - Sie dürfen das Werk bzw. den Inhalt unter folgenden Bedingungen vervielfältigen, verbreiten und öffentlich zugänglich machen sowie Abwandlungen und Bearbeitungen des Werkes bzw. Inhaltes anfertigen: Sie müssen den Namen des Autors/Rechteinhabers in der von ihm festgelegten Weise nennen. Dieses Werk bzw. der Inhalt darf nicht für kommerzielle Žwecke verwendet werden. Die neu entstandenen Werke bzw. Inhalte dürfen nur unter Verwendung von Lizenzbedingungen weitergegeben werden, die mit denen dieses Lizenzbedingungen weitergegeben werden,

Mit der Verwendung dieses Dokuments erkennen Sie die Nutzungsbedingungen an.

\section{Terms of use}

This document is published under following Creative Commons-License: http://creativecommons.org/licenses/by-nc-sa/4.0/deed.en - You may copy, distribute and transmit, adapt or exhibit the work in the public and alter, transform or change this work as long as you attribute the work in the manner specified by the author or licensor. You are not allowed to make commercial use of the work. If you alter, transform, or change this work in any way, you may distribute the resulting work only under this or a comparable license.

By using this particular document, you accept the above-stated conditions of

\section{Kontakt / Contact:}

\section{DeDOCS}

DIPF | Leibniz-Institut für Bildungsforschung und Bildungsinformation Informationszentrum (IZ) Bildung

E-Mail: pedocs@dipf.de

Internet: www.pedocs.de

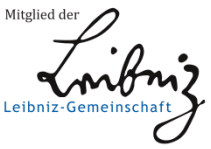




\section{Lernen und Studieren in Lernwerkstätten}

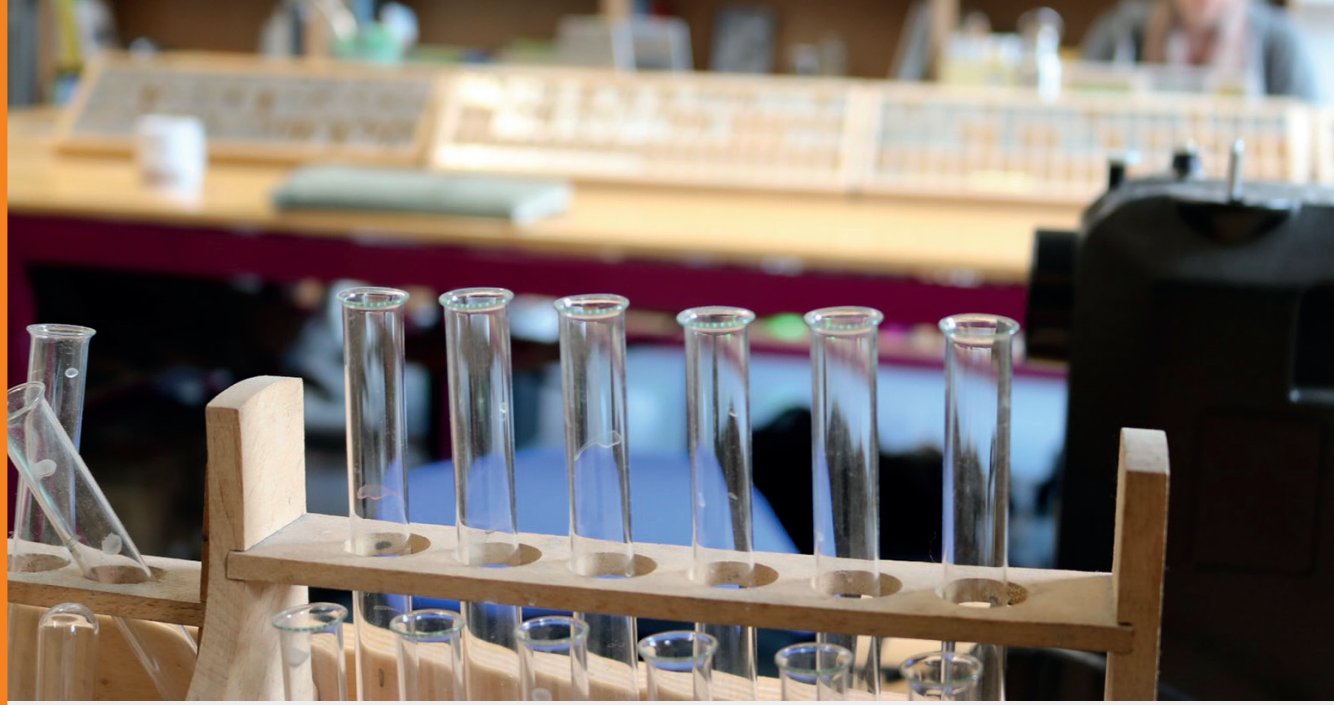

Kathrin Kramer / Dietlinde Rumpf / Miriam Schöps / Stephanie Winter (Hrsg.)

Hochschullernwerkstätten - Elemente von Hochschulentwicklung?

Ein Rückblick auf 15 Jahre Hochschullernwerkstatt in Halle und andernorts 


\section{Kramer / Rumpf / Schöps / Winter Hochschullernwerkstätten - Elemente von Hochschulentwicklung?}




\section{Lernen und Studieren in Lernwerkstätten}

Impulse für Theorie und Praxis

Herausgegeben von

Eva-Kristina Franz, Johannes Gunzenreiner, Barbara Müller-Naendrup, Hartmut Wedekind und Markus Peschel 


\section{Kathrin Kramer \\ Dietlinde Rumpf \\ Miriam Schöps \\ Stephanie Winter \\ (Hrsg.)}

\section{Hochschullernwerkstätten - Elemente von Hochschulentwicklung?}

Ein Rückblick auf 15 Jahre Hochschullernwerkstatt in Halle und andernorts 
Der vorliegende Band ist anlässlich einer Tagung des 15jährigen Bestehens der Hochschullernwerkstatt Erziehungswissenschaften in Halle im November 2020 entstanden.

Gefördert wurden die Tagung und der Band vom Zentrum für Lehrer *innenbildung, der Universitäts- und Landesbibliothek Sachsen-Anhalt sowie der Martin-Luther-Universität Halle-Wittenberg.

Dieser Titel wurde in das Programm des Verlages mittels eines Peer-Review-Verfahrens aufgenommen. Für weitere Informationen siehe www.klinkhardt.de.

Bibliografische Information der Deutschen Nationalbibliothek Die Deutsche Nationalbibliothek verzeichnet diese Publikation in der Deutschen Nationalbibliografie; detaillierte bibliografische Daten sind im Internet abrufbar über http://dnb.d-nb.de.

2020.k. (C) by Julius Klinkhardt.

Coverfoto: (C) Florian Johnke-Liese / Hochschullernwerkstatt.

Druck und Bindung: AZ Druck und Datentechnik, Kempten.

Printed in Germany 2020.

Gedruckt auf chlorfrei gebleichtem alterungsbeständigem Papier.

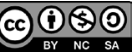

Die Publikation (mit Ausnahme aller Fotos, Grafiken und Abbildungen) ist veröffentlicht unter der Creative Commons-Lizenz: CC BY-NC-SA 4.0 International https://creativecommons.org/licenses/by-nc-sa/4.0/ 


\section{Inhalt}

Kathrin Kramer, Dietlinde Rumpf, Miriam Schöps und Stephanie Winter Einführung in den Band

\section{Teil 1: Historische Entwicklung}

Miriam Schöps und Dietlinde Rumpf

Universität mit Lernwerkstatt in Entwicklung -

Von der Materialausleihe zur Hochschuldidaktik

Hartmut Wenzel

Lernwerkstätten auch für die Sekundarstufen -

Zur Entwicklung der Hochschullernwerkstatt in Halle

Ulrike Stadler-Altmann, Susanne Schumacher, Enrico A. Emili,

Gerda Winkler und Elisabeth Dalla Torre

Hochschullernwerkstätten als Spielball der Bildungspolitik?

Die EduSpace Lernwerkstatt in der Südtiroler Lehrer*innenbildung zwischen nationalen und regionalen Bildungsinteressen

Hartmut Wedekind und Corinna Schmude

Von der Idee zum strukturell verankerten hochschuldidaktischen Prinzip -

Lernwerkstätten und Lernwerkstattarbeit im Studiengang „Erziehung und Bildung in der Kindheit" an der Alice Salomon Hochschule Berlin 68

\section{Teil 2: Wahrnehmung, Selbstverständnis, Einflüsse}

Dietlinde Rumpf und Corinna Schmude

NeHle - Internationales Netzwerk der Hochschullernwerkstätten -

Entwicklungsphasen einer Interessenvertretung und

eines gemeinsamen Begriffsverständnisses

Constantin Beyer und Florian Johnke-Liese

Hochschullernwerkstatt goes digital!

Alexandra und Michael Ritter

Drucken wie ,von gestern' in der Schule der Zukunft -

Die Schuldruckerei als Erfahrungsraum für Kinder und Studierende 
Livia Enders (geb. Makrinus) und Stephanie Winter

Studierende und Dozierende im Spannungsfeld zwischen

Theorie und Praxis - Die Hochschullernwerkstatt als Forschungsraum

John Marcus Sommer

Selbstwahrnehmung in der Hochschullernwerkstatt -

Wissenschaft zwischen Abstraktion und emotionalem Lernen

Lena Kliebe

Hochschullernwerkstatt als Raum und pädagogische Konzeption -

„Mein eigener Lernprozess“

Jerome Kampe

Geschichtsunterricht in der Lernwerkstatt!?

\section{Teil 3: Partizipation und Inklusion}

Melanie Schlag

Hochschullernwerkstatt schlägt Schlager

Kathrin Kramer

Nichtreformistische Reformen - Die Bedeutung von

Hochschullernwerkstätten auf dem Weg zu einer inklusiven Universität am Beispiel des Budgets für Arbeit

Johanna Ingenerf und Pascal Kurz

Menschenrechtsbasierte Bildung im internationalen Dialog -

Ein selbstorganisiertes Seminar mit Austausch

Ines Boban und Andreas Hinz

Hochschullernwerkstatt Halle als Möglichkeitsort -

Von WIRkstatt-Impulsen für inklusive Prozesse

Brigitte Kottmann und Alena Lensker (geb. Beckmann)

Die Lernwerkstatt und der Studiengang „Integrierte Sonderpädagogik“

an der Universität Bielefeld: Impulse, Synergien und Reflexionen

Sarah Dannemann, Tjark Neugebauer, Claudia Schomaker und Rolf Werning

Die LeibnizLernlandschaft: Diversität und Digitalisierung $\left(\mathrm{L}^{2} \mathrm{D}^{2}\right)$

gestalten - Konzeptionelle Gedanken für eine inklusive

Hochschullernwerkstatt an der Leibniz Universität Hannover 


\section{Teil 4: Reflexivität und Professionalisierung}

Kira Wybierek

Praxissemester in der Hochschullernwerkstatt -

Reflexion eines Lernprozesses

Eva Hoffart

„...da man lernt, eigene Gedanken und Ideen noch einmal zu vertiefen“ -

Theoretische Überlegungen und praktische Umsetzungen zum Reflektieren

von Lehramtsstudierenden

Edita Jung und Lena S. Kaiser

„Vielleicht romantisiere ich die Räume meiner Kindheit.“

Zugänge zu einer biographischen Reflexivität in

kindheitspädagogischen Hochschullernwerkstätten

Rolf-Torsten Kramer

Zum Problem der Professionalisierung im Lehramtsstudium und

zum Potenzial der Hochschullernwerkstatt

Mark Weißhaupt, Kathleen Panitz und Elke Hildebrandt

Die Inszenierung von „Theorie und Praxis“ sowie

„Neugier und Desinteresse“ bei der Professionalisierung

angehender Lehrpersonen in Hochschullernwerkstätten

Markus Peschel und Pascal Kihm

Hochschullernwerkstätten - Rollen, Rollenverständnisse und

Rollenaushandlungen

David Paulus, Patrick Gollub und Marcel Veber

Forschendes Lernen und Kasuistik: Überschneidungen und

Abgrenzungen bezogen auf Reflexivität in der

Hochschullernwerkstattarbeit

Pascal Kihm, Jenny Diener und Markus Peschel

Qualifizierungsprozesse und Qualifikationsarbeiten in

Hochschullernwerkstätten - Forschende Entwicklung einer

innovativen Didaktik 
10| Inhaltsverzeichnis

\section{Teil 5: Kooperation}

Georg Breidenstein, Sara Burkhardt, Thorid Rabe und Miriam Schöps

Zur Materialität des Lernens -

Anregungen aus einem interdisziplinären Forum

in der Hochschullernwerkstatt

Miriam Asmus, Kati Friebe, Mirjam Lewin und Kati Misselwitz

Entdeckendes Lernen und Digitale Medien - Ein Traumpaar .

Siglinde Spuller

Kooperation und Kooperatives Lernen als Prinzip

Hochschullernwerkstätten-adäquaten Lernens?

Eine konzeptionelle Verortung

Linda Balzer

Der Effekt selbstregulierenden und forschenden Lernens auf kooperative und individuelle Lernprozesse in der

Lernwerkstatt Religion Plural (LeRP)

Martin Lindner

Werkstattarbeit in der Biologiedidaktik -

Umstellung eines Praktikums auf Projektarbeit

Autor*innenbeschreibungen 
Linda Balzer

\section{Der Effekt Selbstregulierten und Forschenden Lernens auf kooperative und individuelle Lernprozesse in der Lernwerkstatt Religion Plural (LeRP)}

\section{Zusammenfassung}

„Lernen ist ein Prozeß, durch den ein Organismus sein Verhalten als Resultat von Erfahrung ändert. "(CAGE \& BERLINER 1996, 231) Ein Lernprozess bedeutet folglich, dass das Lernen Zeit und ein Ziel braucht. In Lernwerkstätten steht der eigene Lernprozess des*der Lernenden im Fokus. Das Lernen gelingt demnach dann, wenn es von Lernenden selbst ausgeht. Eine Lernwerkstatt bietet somit Lernenden die Möglichkeit, ihr selbstgesteuertes Lernen zu fördern. Dementsprechend können eigene Ziele sowohl organisatorisch als auch inhaltlich effektiv entwickelt und reflektiert werden.

Die Hochschullernwerkstatt Lernwerkstatt Religion Plural (LeRP) unterstützt diese Prozesse durch Selbstreguliertes und Forschendes Lernen. Die LeRP orientiert sich dabei am Modell von Barry J. ZIMMERMAN (2000), das Selbstreguliertes Lernen als eine prozessbezogene Lernschleife präsentiert. Insbesondere wird dabei der Fokus auf die letzte Stufe der Lernschleife gelegt, die die Reflexion vorangegangener Handlungen in Bezug auf zukünftige Handlungen betont. Im Kontext zum Forschenden Lernen ist das Verfahren nach Karin REIBER (2007) maßgeblich. Sie gibt in Einzelschritten vor, wie Forschendes Lernen zielführend angewandt werden kann.

Für die LeRP steht die Entwicklung einer erweiterten und intensiven Reflexionsfähigkeit im Zentrum, die die Professionalisierung der eigenen Handlungskompetenz fokussiert. Während der Praxisphasen, die vor Ort in der LeRP gestaltet werden, sind Einzelund Gruppenarbeiten je nach Bedürfnis frei wählbar. Dieser Aufsatz beschäftigt sich nun damit zu hinterfragen, wie sich Selbstreguliertes und Forschendes Lernen in den jeweiligen Praxisphasen auswirkt. Die Reflexion individueller und gemeinsamer Lernmomente steht somit im Vordergrund, um theoretische Ideen für die Schulpraxis sichtbar zu machen. Für die religionspädagogische Lernwerkstättenlandschaft nimmt die LeRP vor dem Hintergrund der beiden genannten Lerntheorien eine bislang unikale Position ein. 


\section{Eckpunkte der Lernwerkstatt Religion Plural an der Universität des Saarlandes}

Die Lernwerkstatt Religion Plural existiert seit 2016 an der Universität des Saarlandes. Sie wird von der Fachrichtung Katholische Theologie angeboten und kann von Studierenden aller Studiengänge der Lehramtsausbildung besucht werden. Die Hochschullernwerkstatt verfügt über mehrere Räume, darunter eine Art Foyer für theaterpädagogische Übungen und einen Medienraum, die den Studierenden für Lernphasen zur Verfügung stehen. Die LeRP ist eine hochschuldidaktische Lernwerkstatt, die sich das Ziel gesetzt hat, die Professionalisierung der Studierenden zu unterstützen. Sowohl die LeRP als auch sieben weitere Lernwerkstätten konnten im Rahmen des Projekts SaLUt („Optimierung der saarländischen Lehrer*innenausbildung: Förderung des Umgangs mit Heterogenität und Individualisierung im Unterricht ${ }^{\text {"1 }}$ ) aufgebaut werden. SaLUt ist ein Verbundvorhaben der Universität des Saarlandes, der Hochschule der Bildenden Künste Saar und der Hochschule für Musik Saar. ${ }^{2}$ Das Projekt wird im Rahmen der Qualitätsoffensive Lehrer*innenbildung aus Mitteln des Bundesministeriums für Bildung und Forschung gefördert. Die LeRP ist somit umgeben von verschiedenen Hochschullernwerkstätten, die auf dem Campus Saarbrücken vorzufinden sind. Eine Kooperation mehrerer Hochschullernwerkstätten in Lehre und Forschung ist daher nicht unüblich. Somit konnte die LeRP einen engeren Kontakt zu den Bildungswissenschaften und deren Lernwerkstatt herstellen, was den Fokus auf Selbstreguliertes und Forschendes Lernen in der LeRP unterstützte.

\section{Begründungen zum Selbstregulierten und Forschenden Lernen}

In einer schnelllebigen und wissenskonzentrierten Gesellschaft erscheinen gängige Vermittlungsformen, Wissen zu transportieren, oftmals überholt (vgl. FrIEDRICH \& Mandl 1997, 237). Um komplexe Inhalte besser verstehen und strukturieren zu können, benötigt der*die Lernende bestimmte Fähigkeiten der Selbstorganisation. Hierbei tritt das Selbstregulierte, synonym auch als Selbstorganisiertes oder Selbstgesteuertes Lernen verstanden, in den Vordergrund (vgl. Rosendahl 2010, 17). Selbstreguliertes Lernen basiert auf dem Konzept der Selbstregulation. Es unterstützt die Lernenden dabei, Lernvorgänge beliebig oft zu regulieren und dementsprechende Konsequenzen für nachfolgende Handlungen zu treffen (vgl. Zimmerman 2000, 14). Das Arbeiten im Team birgt dabei besondere Herausforderungen, indem verschiedene, manchmal konträre Meinungen berücksich-

$1 \mathrm{https} / /$ www.uni-saarland.de/page/salut/start.html (zuletzt eingesehen am 12.1.20).

2 Vgl. https://www.uni-saarland.de/page/salut/start.html (zuletzt eingesehen am 12.1.20). 
tigt werden müssen. Dabei können „Kollegen, Vorgesetzte [...] wertvolle Quellen für Lernprozesse sein “ (Sonntag \& STEgmaier 2007, 45). Damit die Lernenden selbstreguliert lernen können, sind bestimmte Komponenten zu berücksichtigen, die dem Selbstregulierten Lernen zugrunde liegen: die motivationale, kognitive und metakognitive Komponente. Die motivationale Komponente bezieht sich auf alle Elemente der Motivation, bspw. auch die Selbstmotivierung bei Nichterreichen eines Ziels (vgl. Berg 2006, 16). Die kognitive Komponente hingegen verweist auf die Bereiche der Wissensverarbeitung, so auch auf das Wissen, wie eine bestimmte Aufgabe zu lösen ist (vgl. ebd.). Als Letztes ist die metakognitive Komponente zu nennen, die den Bereich der Selbstbeobachtung, Planung und Anpassung an das Lernziel umschreibt (vgl. Perels 2006, 5). Selbstreguliertes Lernen läuft - angelehnt an Barry J. Zimmerman's Phasenmodell - in folgenden Etappen ab: in einer Planungsphase, Handlungsphase und Reflexionsphase (vgl. Zimmerman 2000, 14). Zur Kontrolle erhalten die Studierenden in der LeRP ein Lerntagebuch, das zum Dokumentieren dieser Schritte dient. Es wurde eigens für die LeRP hergestellt. Selbstreguliertes Lernen erfolgt in Form einer Art Lernschleife, die einen Ist-Zustand und einen Soll-Zustand beinhaltet. Das Ziel der Lernenden besteht darin, den Soll-Zustand zu erreichen. Hierfür ist zu Beginn eine konkrete Zielformulierung notwendig. Deren einzelne Schritte werden individuell im Lerntagebuch festgehalten und nach Bedarf anschließend gemeinsam mit der Dozentin ausgewertet.

Das Abwägen verschiedener Meinungen sowie das Suchen und Finden nach neuesten Erkenntnissen führen in diesem Kontext zum Forschenden Lernen. Im praktischen Fall bedeutet dies in der LeRP, dass die Studierenden auf einen Impuls (z. B. „Außerschulische - interreligiöse - Lernorte") durch die Dozentin Inhalte, Methoden, Konzepte dazu zunächst wahllos sammeln. Nach und nach filtern die Studierenden die für sie relevanten Ergebnisse und präsentieren diese als Resultat auf den genannten Impuls. Laut Sembill \& SeIfried ist Selbstreguliertes Lernen als "Weiterentwicklung und Ausdifferenzierung des Forschenden Lernens" (SEMBILL \& SEIFRIED 2006, 100) zu verstehen. Forschendes Lernen hat nicht zuletzt seit der Hochschulreform in den 70er Jahren vermehrt an Aufmerksamkeit dazugewonnen. Forschendes Lernen sei „ein maßgebliches hochschuldidaktisches Prinzip“ (Mieg 2017, 15). Auch die Studierendenproteste von 2009 zeigten, dass die Lehre an einer Hochschule mehr forschungsorientiertes und selbstständiges Arbeiten der Studierenden ins Zentrum stellen sollte. Der Blick auf einen autonomen und wissbegierigen Lernhabitus rückte somit stärker in den Fokus (vgl. Schelhowe 2013, 14). Karin Reiber gibt konkrete Hinweise, wie Lernarrangements zum Forschenden Lernen gestaltet werden können. In ihrer Ausgangssituation geht sie davon aus, dass die eigene Problemstellung einen subjektiven Bezug zum Lernenden haben sollte. Davon ausgehend soll ein Plan (Ausgangssituation bis hin zur Zielformulierung) erstellt werden, wie der Forschungsprozess in den 
Der Effekt Selbstregulierten und Forschenden Lernens

371

Einzelphasen auszusehen hat. REIBER betont dabei die sozial kontextuierten Rahmenbedingungen, die Raum für Diskussionen geben sollen (REIBER 2007, 9-11). In der Lernwerkstatt wird diese Phase vor allem in Gruppen- und Plenumsgesprächen realisiert.

Die beiden lerntheoretischen Konstrukte sind daher in allen Phasen des Lernens in der LeRP präsent, sowohl in individuellen, als auch kooperativen Lernmomenten.

3 Praxisbeispiele: Selbstreguliertes und Forschendes Lernen in Einzel- und Gruppenarbeit

Selbstreguliertes und Forschendes Lernen sind während der Arbeitszeiten in der LeRP durchgehend präsent. Sowohl für das Arbeiten (eigene, ausgesuchte Lernaufgaben zu einem bestimmten Themengebiet - z. B. Feste im Islam -, gemeinsame Reflexionsgespräche nach vorangegangen gemeinsamen oder individuellen Unterrichtsvorbereitungen usw.) vor Ort als auch in Arbeitssequenzen zuhause wird deutlich, wie die Lernenden lernen. Dabei hat das Wie eine wichtige Funktion. Anhand eines sogenannten Lerntagebuchs reflektieren und protokollieren die Studierenden (zuhause oder in der LeRP) ihren eigenen Lernverlauf, aber auch darüber hinaus Gefühle und Empfindungen, wie das Lernen in der LeRP funktioniert (Abb. 1).

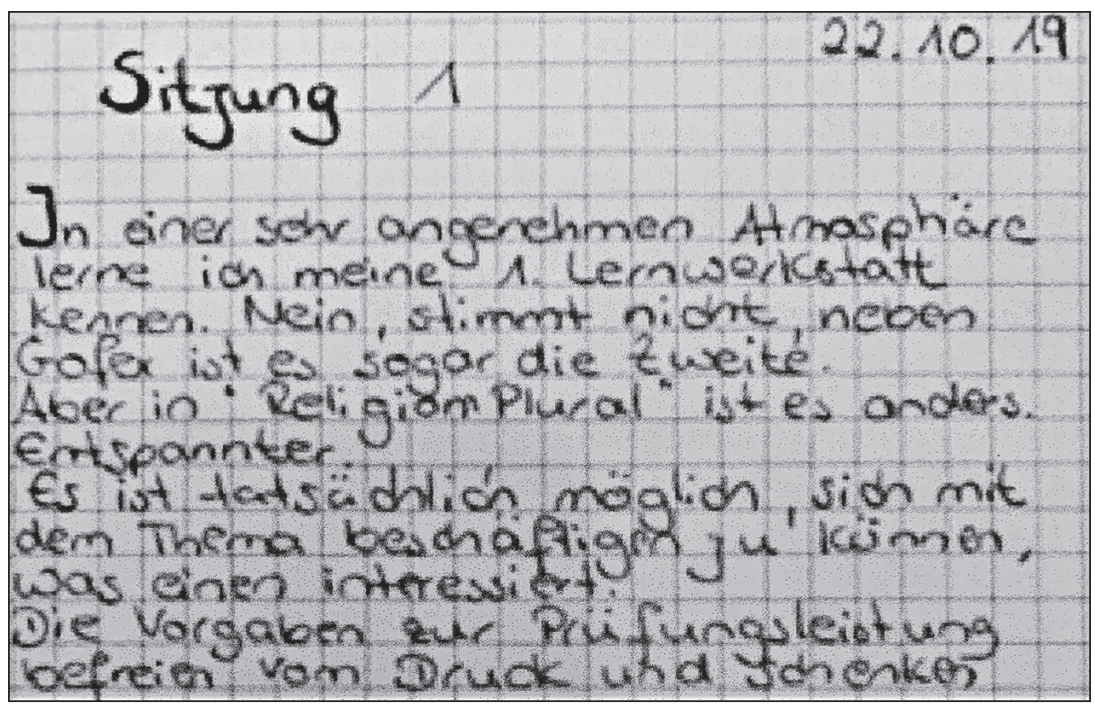

Abb. 1: Auszug aus einem Lerntagebuch

doi.org/10.35468/5858_30 
Das Lerntagebuch wird im Kontext Selbstregulierten Lernens angewandt. Es wird als „Evaluationsinstrument zur Lernprozesskontrolle und zur Lernstandortbestimmung eingesetzt“ (STrauch et al. 2009, 55). Das Lerntagebuch führt „die Lernenden an einen aktiven, selbstreflexiven und eigenverantwortlichen Umgang mit dem eigenen Lernprozess heran“ (ebd.). Während der begleitenden Lernwerkstattzeiten $^{3}$ (ca. 90 Minuten) oder im Anschluss können ihre Ergebnisse individuell mit dem*der Dozierenden besprochen werden. Für die Einzel- und Gruppenarbeiten ergibt sich daher die Frage: Welche Bedeutung hat Selbstreguliertes und Forschendes Lernen sowohl in Einzelarbeit als auch in Gruppenarbeit?

\subsection{Einzelarbeit in der LeRP}

Die Studierenden dürfen in der LeRP grundsätzlich jene Sozialform für die Bearbeitung einer Aufgabe wählen, die für sie am sinnvollsten erscheint. Dieses Vorgehen basiert auf dem Prinzip des individuellen Lernwerkstatt-Verständnisses der LeRP und orientiert sich hiermit am offiziellen Positionspapier des Verbunds europäischer Lernwerkstätten. Für die Position der Lernenden wird festgehalten, dass sie „selbst das jeweilige Maß an individuellem und gemeinsamem Arbeiten“ (VELW 2009, 7) bestimmen. Dabei wird jede*r als „Subjekt wahrgenommen und respektiert" (VELW 2009, 7).

Für bestimmte Aufgaben ist Einzelarbeit in der LeRP besonders geeignet. Zum einen ist sie sinnvoll, wenn die eigene, subjektive Reflexionskompetenz zu einem bestimmten Thema geschult werden soll. Zum anderen kann sie sich unterstützend auf die eigene Lehrkompetenz als zukünftige*r Lehrer*in auswirken, indem Unterrichtskonzepte in einer bestimmten Zeit allein erstellt werden. Um dies genauer zu beleuchten, soll vor dem Hintergrund Selbstregulierten und Forschenden Lernens ein Beispiel erläutert werden. Für eine fiktive Unterrichtsgestaltung durften die Studierenden einzeln einen religiösen Gegenstand aus einer verdeckten Tasche entnehmen. Das Ziel bestand darin, den Gegenstand in eine Unterrichtsgestaltung für die jeweilige Schulform einzubinden. Der jeweilige Gegenstand musste von jedem*r Studierenden selbst erkannt und benannt werden. Die Studierenden befanden sich hiermit in der Lernschleife des Selbstregulierten Lernens (Planungsphase - Handlungsphase - Reflexionsphase). In der Ausgangssituation wurde der Ist-Zustand benannt: ich als Lernende*r habe einen religiösen Gegenstand bekommen. Der Soll-Zustand lautete: es muss eine Unterrichtsstunde erstellt werden, in der der religiöse Gegenstand sinnvoll integriert werden kann. Dabei war zu beobachten, dass einige der Studierenden Probleme hatten

3 Die Studierenden können die Hochschullernwerkstatt zu den Öffnungszeiten besuchen. Gleichzeitig werden von der Dozierenden sogenannte begleitete Seminarsitzungen zu bestimmten Themen angeboten, die von allen Lehramtsstudierenden besucht werden können, d. h. die Dozierende ist während der gesamten Bearbeitungszeit als Lernbegleiterin vor Ort. 
herauszufinden, wie der Gegenstand überhaupt heißt oder was er bedeutet. In solchen Situationen wird die motivationale Komponente Selbstregulierten Lernens deutlich. Je schwieriger oder unbekannter der Gegenstand war, desto mehr wirkte sich dieser Umstand auf die Motivation aus. Die Studierenden durften sich jederzeit an die Dozierende wenden, die in solchen Momenten hauptsächlich als Lernbegleiterin fungiert. Kleinere Hinweise der Dozierenden führten somit dazu, dass sich der*die Lernende engagierter dem religiösen Gegenstand widmete. Bereits vorhandenes Wissen konnte hierbei mit neuem Wissen verknüpft werden (kognitive Komponente). Während ihrer individuellen Lernphasen wurden die Lernwege und -umwege detailliert festgehalten. Für eine fiktive Schulstunde wollte ein Studierender das Thema Torarolle behandeln. Ihm war es wichtig, dass Schüler*innen einen subjektiven Bezug zu diesem Gegenstand bekommen (korrelative Religionsdidaktik): Dabei kam ihm der Gedanke, dass die Schüler*innen selbst eine Torarolle anfertigen könnten, damit sie jederzeit, auch haptisch, für jede*n Einzelne*n verfügbar sei. Seine Idee basierte darauf, dass sich die Schüler*innen Gedanken darüber machen sollten, was in ihrem Leben wichtig und heilig sein könnte, um es in die eigene Torarolle zu schreiben. Im Judentum ist die Tora Grundlage für das jüdische Leben (Essensvorschriften, Gebete usw.). Sie wird als heilige Schrift bezeichnet und hat folglich einen zentralen Platz im Leben der Juden. Der Studierende wollte hiermit aufzeigen, wie der Religionsunterricht lebensnah und schüler*innenorientiert gestaltet werden und gleichzeitig kreative und ästhetische Elemente beinhalten kann.

Das Lerntagebuch unterstützte die Lernenden dabei, die Ziele weiterhin zu verfolgen. Es erfolgte somit eine Selbstbeobachtung (Self-Monitoring) des Lernverlaufs, wodurch die metakognitive Komponente berücksichtigt wurde. Im letzten Teil der Bearbeitung (Reflexionsphase) nahm sich jede*r Lernende Zeit, die vorangegangene Arbeitsphase zu reflektieren. Der individuelle Lernweg und die damit verbundene Zielorientierung, den Unterricht mit einem religiösen Gegenstand didaktisch aufzubereiten, wurde nochmals besprochen. Die Lernenden sollen in dieser abschließenden Reflexion Erkenntnisse (auch eine gewisse Selbstbewertung) über ihren Lernverlauf gewinnen, damit sie in ähnlichen Arbeitsphasen effektiver arbeiten und gegebenenfalls bestimmte Störfaktoren (z. B. Ablenken durch andere Gruppen) bereits zu Beginn vermeiden können. Gleichzeitig konnten bestimmte Strategien entworfen werden, auf die für zukünftige Aufgaben immer wieder Bezug genommen werden kann.

Jede*r Studierende arbeitete von Anfang an in einem forschend-entdeckenden Umfeld. Durch das Entnehmen eines verdeckten und unbekannten Gegenstandes wurde jede*r Einzelne in ihrer*seiner Neugierde geweckt. Neben Studierenden, die teils Schwierigkeiten hatten, den Gegenstand zu erkennen, gab es auch eifrige Studierende, die von Anfang an sehr motiviert waren. Jede*r von ihnen bemühte sich um eine ausführliche Ausarbeitung hinsichtlich Beschreibung und Funktion 
des Gegenstandes für die jeweilige Religion. Mit einem gut ausgearbeiteten Inhaltskonzept konnte die Schulstunde konkret ausgearbeitet werden. Diesen Lernenden eröffneten sich dadurch mehr Möglichkeiten als den anderen, da sie durch ihr konzentriertes „Mehrwissen“ über den Gegenstand variable Einsatzmöglichkeiten für den Unterricht parat hatten. Die freiwillige und schon fast unbegrenzte Erkundungsphase über den religiösen Gegenstand ist bei den eifrigen Lernenden mit einer ausgeprägten intrinsischen Motivation zu begründen. Jede*r konnte mit dem religiösen Gegenstand auf seine*ihre eigene Art und Weise arbeiten. Diese Freiheit begünstigte ein Lernen im eigenen Interesse, womit eine „persönliche Bedeutsamkeit von Lernprozessen“ (Hiebl 2014, 55) einherging.

\subsection{Kooperatives Lernen bzw. Gruppenarbeit in der LeRP}

Auch das kooperative Lernen bzw. das gemeinsame Lernen in der Gruppe ist in der LeRP eine regelmäßig angewandte Sozialform. Die Studierenden arbeiten innerhalb der Lernwerkstatträume häufig an einem Ort, wo sie sich wohlfühlen. Diese Rückzugsmöglichkeit ist besonders für Gruppen sinnvoll, da ein gemeinsamer Austausch von konstruktiven Gesprächen begleitet wird und daher die Atmosphäre sehr lebendig sein kann. Ein Beispiel aus der Gruppenarbeitsphase ist die Planung eines außerschulischen Lernangebotes. Diese Thematik ist häufig zentraler Gegenstand in Gruppenarbeiten, da sie viel Zeit erfordert und die Gruppendynamik einen sichtbaren Multiplikator für ein gelingendes Konzept darstellt. Für das nun angeführte Exempel wurde eine konkrete Gruppe durch die Dozierende beobachtet. Andere Gruppenarbeiten fanden zeitgleich zum selben Thema statt. Die Beobachtung des Lernverlaufs und der -fortschritte innerhalb einer Gruppenphase sind sehr spannend, da viele einzelne Ideen zu einem Geflecht zusammenlaufen. Auch für das ausgewählte Beispiel wurde eine Zielformulierung zu Beginn gemeinsam aufgestellt. Diese gab den Soll-Zustand wieder, nämlich ein außerschulisches Lernangebot zu planen. Der Soll-Zustand konnte im weiteren Verlauf konkretisiert werden, indem genauer gesagt wurde, was der außerschulische Lernort leisten soll. Grundsätzlich war zu beobachten, dass die Planungsphase von sehr vielen, unterschiedlichen und manchmal utopischen Vorstellungen (z. B. mit einer Grundschulklasse eine KZ-Gedenkstätte besuchen) bezüglich der geographischen Lage des außerschulischen Lernortes gekennzeichnet war. Hier spielte die motivationale Komponente erneut eine wichtige Rolle. Gemeinsam musste die Motivation aufrecht erhalten bleiben, am bereits begonnen Konzept - trotz einiger organisatorischer Hindernisse - weiterzuarbeiten. Abhilfe konnte hier ein gemeinsam erstelltes Denkprotokoll leisten, das beliebig oft korrigiert und erweitert werden kann. Im Gegensatz zum Lerntagebuch, das für jeden Einzelnen gilt und diskret behandelt wird, ist ein Denkprotokoll in Gruppenarbeiten oftmals sinnvoll für das Sammeln von Impulsen, unsortierten Meinungen usw. Es dient der Strukturierung mehrerer Ideen und Vorschläge (vgl. Landmann 2009, 
61), die zunächst wahllos gesammelt werden. Diese Ergebnisse können dann für die gestellte Aufgabe verwendet werden (s.o.). Auch in dieser Arbeitsphase war das Denkprotokoll ein wichtiges Element, in dem die Studierenden visuell anhand ihrer Notizen erkannt haben, dass tatsächlich nur ein oder zwei Lernorte in Frage kamen. Hier zeigt sich der Vorteil im kooperativen Lernen. Man wägt gemeinsam ab, welche Aspekte tatsächlich sinnvoll und wichtig sind. Für Selbstregulierte Lernprozesse kann kooperatives Lernen zusätzlich einen positiven Effekt auf das Lernverhalten und die Motivation der gesamten Gruppe aufweisen, indem „in der Auseinandersetzung mit anderen Personen [...] Unklarheiten beseitigt, Wissen vertieft und neue Bedeutungen des Lerninhalts erschlossen werden. [...] Die Interaktion mehrever Personen mit unterschiedlichem Wissensstand eröffnet gute Chance[n,] den Wissenserwerb zu fördern" (KonRAd 2008, 99).

Als schließlich ein geeigneter Lernort gefunden werden konnte, wurde die Handlungsphase von Ideen und Aufgabenstellungen der Studierenden begleitet, die von den Schüler*innen vor Ort bearbeitet werden sollten. In der Beobachtung dieser Gruppe wurde deutlich, wer bereits Vorerfahrungen mit Planungen für auBerschulische Lernorte hatte (kognitive Komponente). Vorerfahrungen konnten somit sinnvoll mit neuen, innovativen Ideen verknüpft werden. Diejenigen, die darin weniger bewandert waren, konnten ihren Wissensfundus dementsprechend ergänzen. Es war deutlich zu erkennen, dass eine Gruppendynamik vorhanden war, die regelmäßig ihre Zielformulierung kontrollierte (metakognitive Komponente). Beispielsweise wurden Aufgabenformulierungen für das Lernen vor Ort korrigiert oder potenzielle Störfaktoren wurden notiert, damit der Ablauf und das Arbeiten am Lernort und das eigentliche Lernziel im Fokus behalten werden kann.

In der Schlussphase stellte die Gruppe ihr Konzept im Plenum vor. Dabei ist es grundsätzlich wichtig, in einen konstruktiven und respektvollen Austausch zu treten. Die LeRP verfolgt hierbei einen sehr kollegialen und gemeinschaftlichen Umgang hinsichtlich Kritik und gemeinsamen Verbesserungen. Somit konnte eine Reflexion über das erstellte Konzept und die Gruppenarbeit erfolgen. In diesem Schritt konnten sich die Gruppenmitglieder auch einzeln dazu äußern, welche Arbeitsphasen ihnen besonders Spaß gemacht haben und wo sie evtl. noch Wissen individuell ergänzen konnten. Auch in ihrem Denkprotokoll wurden einzelne Überlegungen nochmals festgehalten (Abb. 2): 
- Eventuelle Veränderungen im Nachhinein?

o Anderer Ort zum Besprechen als Saar-Treppe, um die Schwere dieses Ereignisses deutlicher zu machen.

o Noch mehr Wert auf Kommunikation legen, um den Kindern nicht nur die Unterschiede deutlich zu machen, sondern gezielt auf Ereignis hinzulenken.

Abb. 2: Auszug Denkprotokoll

Forschendes Lernen war demnach auch in der Gruppenarbeit zu sehen. Die Studierenden mussten zunächst einen geeigneten Lernort suchen und ihn nach Funktion und auf Verwertbarkeit für die Schüler*innen hin untersuchen. Der Beginn war daher durch eine sehr intensive Arbeitsphase gekennzeichnet, da zunächst viele verschiedene Orte gesammelt und beleuchtet wurden. Für das Fach Religion werden häufig die üblichen Gotteshäuser als außerschulische Lernorte besucht (vgl. Köster 2016, 189). Jedoch war es der Gruppe wichtig, ihren Lernort mit einem Erlebnisgefühl in der Natur zu verknüpfen. Meines Erachtens ist dieser Aspekt bereits ein deutliches Indiz für Forschendes Lernen, indem die Studierenden nach ihren eigenen Vorlieben und Interessen gearbeitet haben. Es bleibt festzuhalten, dass durch kooperatives Lernen die Studierenden ihre Gruppendynamik steigern konnten, was dazu führte, dass sie ihre Aufgabe erfolgreich absolvieren konnten. Kooperatives Lernen ist in der Fachdiskussion Selbstregulierten Lernens mittlerweile bekannt und erfährt daher eine vermehrte Anwendung, denn „diese Art des Lernens [sei] häufig anregender als Einzellernen, da durch die unterschiedlichen Ansichten, Vorkenntnisse und Ideen der verschiedenen Gruppenmitglieder eine höhere Kreativität und bessere Leistungen beim Problemlösen erzielt würden“" (Konrad 2008, 100).

\section{Fazit}

Selbstreguliertes und Forschendes Lernen spielen sowohl in Einzelarbeit als auch in Gruppenarbeiten eine wichtige Rolle. Selbstreguliertes Lernen wirkt sich insbesondere unterstützend aus, wenn die eigenen Lernprozesse kontrolliert und reguliert werden müssen. Dies war in Einzelarbeiten in der LeRP intensiv zu beobachten, da sich die Lernenden ausschließlich auf ihre eigenen Lernvorgänge fokussiert haben. Protokolliert wurden diese mittels eines Lerntagebuchs. Dies ermöglicht zugleich für nachfolgende Handlungen Konsequenzen zu ziehen und diese schriftlich im Lerntagebuch festzuhalten.

Im kooperativen Lernen hingegen stand eine gemeinsame Aufgabenbewältigung der Studierenden im Vordergrund. Somit waren individuelle Lernvorgänge weni- 
ger im Fokus der Beobachtung, sondern eher das Zusammenarbeiten der Gruppe, die sich darum bemühte, die Zielfokussierung aufrechtzuerhalten und die Lernprozesse zu regulieren. Die von Beginn an gesammelten Ideen, Vorstellungen und Impulse im Denkprotokoll wurden von allen Studierenden zusammengetragen und ausgewertet. Im kooperativen Lernumfeld konnten die Studierenden ihre Gruppendynamik steigern, was die Bewältigung der Aufgabe z. B. auf motivationaler Ebene vorantrieb.

Des Weiteren war ein forschender Habitus sowohl in Einzelarbeit als auch in der Gruppenarbeit ein wichtiger Aspekt. Individualbasiertes Lernen ermöglichte den Lernenden, experimentell Themen zu behandeln, wodurch bisher unbekannte didaktische - Erkenntnisse (Beispiel Torarolle) bei den Lernenden geweckt werden konnten. Insbesondere zeigte sich dies bei der Erstellung von Unterrichtskonzepten, die einen religiösen Gegenstand zum Thema hatten. Der Lernzuwachs war demnach individuell zu betrachten, indem die Lernenden selbst erkannt haben, was sie inhaltlich ergänzen mussten bzw. woran sie weiterarbeiten wollten.

In der Gruppenarbeit hat das gemeinsame Forschende Lernen dazu beigetragen, die Aufgabe lösen zu können. Es trieb die Gruppe voran, auch bei Umwegen weiter an ihrem Vorhaben festzuhalten. Der Aspekt des Forschens kann folglich „als Strategie zur Bearbeitung komplexer Probleme gesehen werden, wie sie auch im Berufsleben vorkommen, sodass im Zuge einer Beteiligung an Forschung erworbene Problemlösestrategien in die Berufstätigkeit transferiert werden können“" (Fichten 2010, 132).

Für die Hochschullernwerkstatt Lernwerkstatt Religion Plural ist somit festzuhalten, dass sie den Studierenden ein Lernen in einer neuen Lernkultur eröffnet. Im Zuge der Diskussionen um Handlungsoptimierung und Professionalisierung der Reflexionskompetenz sieht sich die LeRP an der richtigen Stelle, mit ihrem Konzept die Lehrer*innenbildung voranzutreiben. Die Lehrer*innenprofessionalisierung kann somit erst recht unterstützt werden, wenn sich die an den Universitäten vorhanden Lehrangebote an Konzepten von Hochschullernwerkstätten orientieren. Diese bieten doch gerade die Grundlage des professionellen pädagogischen Handelns, d. h. es müssen Räume geschaffen werden, die Zeit und Möglichkeiten des Erprobens und Entwickelns fördern. Die LeRP hat dies durch die Vernetzung zu anderen Hochschullernwerkstätten, insbesondere den Bildungswissenschaften der Universität des Saarlandes, erreicht. Sie setzt mit ihrem Konzept neue Akzente für die religionspädagogische Lehrer*innenbildung, indem sie die u. a. oben dargestellten Ansätze des Lehrens und Lernens für Studierende ermöglicht. 


\section{Literatur}

BERG, Christoph (2006): Selbstgesteuertes Lernen im Team. Mit 33 Abbildungen und 8 Checklisten, Heidelberg.

Cage, Nathaniel L. \& Berliner, David C. (1996): Pädagogische Psychologie, 5. Aufl. Weinheim: Psychologie Verlags Union.

Fichten, Wolfgang (2010): Forschendes Lernen in der Lehrerbildung. In: Eberhardt, Ulrike (Hrsg.): Neue Impulse in der Hochschuldidaktik, Sprach- und Literaturwissenschaften. Wiesbaden: VS Verlag für Sozialwissenschaften, Springer Fachmedien, 127-182.

Friedrich, Helmut F. \& Mandl, Heinz (1997): Analyse und Förderung selbstgesteuerten Lernens. In: Weinert, Franz E. \& Mandu, Heinz (Hrsg.): Psychologie der Erwachsenenbildung. Göttingen: Hogrefe, 238-293.

Hiebs, Petra (Hrsg.) (2014): Lernwerkstätten an Schulen: aus der Perspektive von Schulleitern und Schülern. Berlin: Lit.

Köster, Norbert (2016): Historische Orte als außerschulische Lernorte im Religionsunterricht. Perspektiven aus Geschichtsdidaktik, Kirchenraum-, Museums- und Gedenkstättenpädagogik. In: Bork, Stefan \& GärTner, Claudia (Hrsg.): Kirchengeschichtsdidaktik: Verortungen zwischen Religionspädagogik. Stuttgart: Kohlhammer, 188-203.

KonRad, Klaus (Hrsg.) (2008): Erfolgreich selbstgesteuert lernen. Theoretische Grundlagen, Forschungsergebnisse, Impulse für die Praxis. Bad Heilbrunn: Klinkhardt.

Landmann, Meike; Perels, Franziska; Отто, Barbara \& Schmitz, Bernhard (2009): Selbstregulation. In: Wild, Elke \& Möller, Jens (Hrsg.): Pädagogische Psychologie. Heidelberg: Springer, 49-70.

Mieg, Harald A. (2017): Einleitung: Forschendes Lernen - erste Bilanz. In: Mieg, Harald A. \& Lehmann, Judith (Hrsg.): Forschendes Lernen. Wie die Lehre in Universität und Fachhochschule erneuert werden kann. Frankfurt: Campus, 15-36.

Perels, Franziska (Hrsg.) (2006): Selbstreguliertes Lernen. Wiesbaden: IQ kompakt.

Reiber, Karin (2007): Forschendes Lernen als Leitprinzip zeitgemäßer Hochschulbildung. In: Tübinger Beiträge zur Hochschuldidaktik Bd. 1, 6-12.

RosendaHL, Johannes (Hrsg.) (2010): Selbstreguliertes Lernen in der dualen Ausbildung: Lerntypen und Bedingungen. Bielefeld: wbv Media.

Schelhowe, Heidi (2013): Zur Einführung: Forschendes Lernen als Profilelement einer Universität. In: Huber, Ludwig; Kröger, Margot \& Schelhowe, Heidi (Hrsg.): Forschendes Lernen als Profilmerkmal einer Universität. Beispiele aus der Universität Bremen. Bielefeld: UVW, 11-20.

Sonntag, Karlheinz \& Stegmaier, Ralf (Hrsg.) (2007): Arbeitsorientiertes Lernen: zur Psychologie der Integration von Lernen und Arbeit. Stuttgart: Kohlhammer.

Strauch, Anne; Jütten, Stefanie \& Mania, Ewelina (Hrsg.) (2009): Kompetenzerfassung in der Weiterbildung: Instrumente und Methoden situativ anwenden. Bielefeld: Bertelsmann.

Vorstand des Verbundes europäischer Lernwerkstätten (Hrsg.) (2009): Positionspapier des Verbunds europäischer Lernwerkstätten (VeLW) e.V. zu Qualitätsmerkmalen von Lernwerkstätten und Lernwerkstattarbeit. Im Internet: https://www.forschendes-lernen.net/files/eightytwenty/materialien/VeLW-Broschuere.pdf.

Zimmerman, Barry J. (2000): Attaining Self-Regulation. A social cognitive perspective. In: BocKAerts, Monique; Pintrich, Paul R. \& Zeidner, Moshe (Hrsg.): Handbook of Self-Regulation. San Diego: Academic Press, 13-39. 